\title{
REPRODUKTION UND POPULATIONSENTWICKLUNG DER PARASITISCHEN NilLBE VARROA JACOBSONI OUD. IN ABHÄNGIGKEIT VOM BRUTZYKLUS IHRES WIRTES APIS MELLIFERA L.
}

\author{
(I. TEIL) \\ Alfred E. SCHULZ \\ Institut für Bienenkunde der Polytechnischen Gesellschaft an der Universität Frankfurt/M. \\ Karl 1 . Frisch-Weg 2 \\ $D-6370$ Oberursel/Ts.
}

ZUSAMMENFASSUNG

Die Reproduktionsquoten von Varroa in Arbeiter- und Drohnenbrut wurden mit durchschnittlich 1,3 fertilen Töchtern in Arbeiter- und 2,6 fertilen Töchtern in Drohnenbrut pro Vermehrungszyklus festgelegt. Die ermittelte Reproduktionsfrequenz ergab, daß $78 \%$ aller Milben einmal, $22 \%$ dagegen zweimal in Eilage gehen. Bei der Präferenz der Drohnen- vor Arbeiterbrut wurde ein Faktor von 8,6 ermittelt. Bei ausreichend vorhandener offener Bienenbrut drangen innerhalb 6 Tagen ca. $44 \%$, innerhalb 12 Tagen $69 \%$ und binnen 24 Tagen $90 \%$ aller reproduktionsfähigen Jungmilben in dic Brutzellen ein. Milben, die bis zum 50. Lebenstag keine Brutzelle aufgesucht haben, scheinen für die Populationsdynamik ohne Belang. Varroa-Weibchen die bereits in Reproduktion waren und zu einem weiteren Vermehrungsgang in Zellen eindrangen, taten dies nahezu unmittelbar nach Schlupt mit der Jungbiene. Bei variablem Brutzellenangebot konnte die direkte Abhängigkeit der Eindringquote reproduktionswilliger Milben von der Menge der verfügbaren Wirtszellen nachgewiesen werden.

\section{EINLEITUNG}

Aufgabe dieser Arbeit war es, einen Beitrag zur Erfassung wesentlicher Daten der Vermehrungsbiologie von Varroa jacobsoni (Oud., 1904) zu leisten, um ein umfassenderes Bild von der Populationsdynamik des Parasiten im Apis mellifera-Volk zu gewinnen. 
Es wurden folgende Fragestellungen untersucht :

1) Vermehrungsrate von Varroa in Arbeiter- und Drohnenbrut.

2) Frequenz der Reproduktionszyklen von Varroa.

3) Zeitpunkt des Eindringens von Varroa in $\not$-Brut unter kontrollierten Bedingungen.

4) Abhängigkeit der Reproduktion des Parasiten vom Brutzyklus des Wirtes unter natürlichen Bedingungen.

5) Attraktivität von Arbeiter- und Drohnenbrut für infektionsbereite Milben.

Die Untersuchungen 1-4 wurden an kontrolliert erzeugten Brutserien durchgeführt. Dazu wurden die Versuchsmagazine mittels $\&$-Absperrgitter in Brutund Pflegeraum unterteilt. Den Königinnen wurden zum Bestiften jeweils 3-4 ausgebaute Leerwaben gegeben, die im Zyklus von 6 Tagen gegen neue ausgetauscht wurden. Die bebrüteten Waben wurden zur weiteren Brutpflege bis zum 18. Entwicklungstag (12-18 Tage alte Brut) in die Brutpflegezarge gestellt. Danach kamen dic Untersuchungswaben in milbendichten Schlupfkästen in Brutschränke bei $34^{\circ} \mathrm{C}$ und ca. $70 \%$ rel. Luftfeuchte.

Bei Bearbeitung der verschiedenen Fragestellungen kamen unterschiedliche Auswertungsmodi zur Anwendung :

\section{1) Vermehrungsrate von Varroa in Arbeiter- und Drohnenbrut}

Zur Diagnose wurde die Brut am 21. Tag nach Einstellung in den Brutraum (15-21 Tage alte Brut) durch Tiefkühlung abgetötet, die Zellen einzeln geöffnet und der Varroabefall registriert. Es wurden lediglich Milben und deren Deutonymphen von Puppen mit dunklen Augen ( $\mathrm{Pd} / \mathrm{Pdl}$ ) erfaßt, da ausschließlich sie mit der adulten Biene geschlüpft wären. Die Altersbestimmung der Bienenbrut erfolgte nach JAY (1962). Es wurden Erhebungen gemacht an : 155 markierten und 450 unmarkierten Varroae in $\Varangle$-Brut, sowie an 660 unmarkierten Milben in $0^{x}$-Brut.

\section{2) Frequenz der Reproduktionszyklen von Varroa}

Zur Ermittlung der Reproduktionsfrequenz ließen wir markierte Milben im Brutschrank schlüpfen. 78 Milben wurden ein zweites Mal gekennzeichnet und die «Muttermilben» erneut in die Versuchsvölker gesetzt. Anschließend wurde analog zu 1) verfahren.

\section{3) Zeitpunkt des Eindringens von Varroa in ఫ̧-Brut unter kontrollierten Bedingungen}

Die Untersuchungen zur Frage mit welcher zeitlichen Verteilung VarroaMilben zur Reproduktion $\Varangle-B r u t z e l l e n$ aufsuchen, wurden mit verschiedenfarbig 
markierten Milben durchgeführt. Es wurde davon ausgegangen, daß bei geringer Varroa-Befallsdichte $(<1 \%)$ alle reproduktionswilligen Milben-Weibchen eine geeignete Brutzelle finden konnten. Zur Rekonstruktion des Eindringtermins wurden die unter kontrollierten Bedingungen erzeugten Brutwaben am 21. Entwicklungstag (15-21 Tage alte Brut) abgetötet und zur Auswertung Zelle für Zelle im Labor untersucht.

Die Altersbestimmung der Bienenpuppen erfolgte nach JAY (1962). Von der Probenentnahme wurde der Eindringtermin der Milbe auf den 8. Entwicklungstag der Bienenpuppe (angenommener Zeitpunkt der Infektion, +/- 1 Tag) zurückberechnet und zum Datum des Eindringens der markierten Milben in das Bienenvolk in Beziehung gesetzt.

Untersucht wurden drei Versuchsgruppen :

a) junge Milben $(\mathrm{n}=142)$;

b) Varroa- + nach voraufgegangener Eilage $(\mathrm{n}=43)$;

c) Varroae gemischten Alters $(\mathrm{n}=298)$.

4) Abhängigkeit der Reproduktion des Parasiten vom Brutzyklus des Wirtes unter natürlichen Bedingungen

Die Abhängigkeit der Reproduktion bei Varroa vom Brutzyklus des Bienenvolkes wurde an zwei Versuchsgruppen mit natürlichem Milbenbefall untersucht :
Gruppe a) 15 Völker im Zeitraum Mai-Juli ;
Gruppe b) 4 Völker im Zeitraum August-September.

Gruppe a) wurde über 8 Brutserien, Gruppe b) über 4 Brutserien von je 6 Tagen einer fortgesetzten Brutkontrolle unterzogen. Dazu ließen wir die Bienen serienweise in varroadichten Kästen im Brutschrank (s.o.) schlüpfen. Zur Auswertung wurden die Bienen täglich entnommen und, soweit dies zeitlich möglich war, einzeln auf Varroa-Befall untersucht. Milbenfreie Bienen wurden den Versuchsvölkern wieder zugesetzt. War dies unmöglich, so wurden die Proben tiefgekühlt und zu einem späteren Zeitpunkt der Milbenbefall durch Auswaschen in Benzin (RITTER und RUTTNer, 1980) ermittelt. Die Bestimmung der jeweiligen Bienenmengen erfolgte durch Zählung oder Wägung.

Zur Endauswertung wurden die Völker abgetötet und nach o.g. Methode ausgewaschen. Der Varroa-Ausgangsbefall wurde durch Addition der im Untersuchungszeitraum ermittelten «Muttermilben » (Milbenwerte der einzelnen Brutserien dividiert durch den Faktor 2,3 $=1$ Mutter $+\varnothing 1,3$ Töchter) mit der Anzahl «Restmilben» im Volk bei Versuchsende errechnet. 


\section{5) Attraktivität von Arbeiter- und Drohnenbrut für infektionsbereite Milben}

Im Monat Mai sollte die Präferenz von $\sigma^{\star}$-Brut vor $\Varangle$-Brut an jeweils 13 Stichproben aus Bienenvölkern mit natürlichem Varroa-Befall erfaßt werden. Gezählt wurden nur adulte Milben ( Mütter 》) auf Bienenpuppen der Entwicklungsstadien Pw (Puppe mit weißen Augen) bis Pd (Puppe mit dunklen Augen).

\section{MATERIAL UND METHODE}

Die Untersuchungen der Fragestellungen 1-3 wurden mit individuell markierten Milben durchgeführt. Darüberhinaus wurden Erhebungen an natürlich infizierter Bienenbrut angestellt (1, 4 und 5$)$.

Milben :

Von im Brutschrank frisch geschlüpften Bienen wurden Milben mit Hilfe eines feinen Pinsels abgefangen. Dabei wurden hellsklerotisierte Varroae als Jungmilben klassifiziert. Milben wurden nach vollendeter Eilage aus Brutzellen im Stadium Pd (Puppen mit dunklen Augen) herauspräpariert. Außerdem wurden Varroa-Milben von Stockbienen abgesammelt, Die Zugabe der markierten Milben zum Bienenvolk erfolgte über Jungbienen.

\section{Markierungsmethode :}

Fluoreszenzpigmente (Simon \& Werner, Schwalbach/Ts.) wurden in einen Cyanacrylatkleber (Bostik, Oberursel/Ts.) eingemischt. Mit Hilfe einer Markierungsnadel $(\varnothing 0,1 \mathrm{~mm}$ ) wurde das Farbmedium auf das Dorsalscutellum der unbetäubten Milben appliziert. Es verhielt sich atoxisch, besaß gute Haltbarkeit und erwies sich hinsichtlich der Identifizierbarkeit der markierten Versuchstiere - bei der Kleinheit der Markierungen ( $\varnothing 0,2 \mathrm{~mm})$ - als vorteilhaft.

In Käfigversuchen zeigten markierte Milben verglichen mit unmarkierten keinen Unterschied in der Lebensdauer. Dennoch deuten die im Versuch beobachteten Ausfälle an, daß die Behandlung gavierende Folgen hatte : Von den insgesamt 1323 markiert eingesetzten Milben wurden 666 (50\%) vermißt; $1979 / 1981$ starben von den in Bienenzellen eingedrungenen Varroae $40 \% ; 1982$ waren von den eingedrungenen $61 \%$ nicht in Eilage gegangen. Schädigungen der Milben beim Ablesen von den Bienen bzw. beim Zeichenvorgang, sowie Verluste über Flugbienen sind ais Ursache anzunehmen. Zur Absicherung der Ergebnisse wurden weitere Untersuchungen an natürlich infizierter Bienenbrut durchgeführt.

Bienen und Bienenhaltung :

Die Untersuchungen wurden an Carnica-Völkern mit jungen Königinnen durchgeführt. Die Völker waren in Freilandflugzelten, sowie freifliegend aufgestellt.

Die Stichprobenauswahl erfolgte zufällig, wobei ausschließlịch Völker mit geschlossenem Brutnest (mehr als 500 verdeckelte Zellen) einbezogen wurden.

\section{ERGEBNISSE}

1) Vermehrungsrate von Varroa in Arbeiter- und Drohnenbrut

Sämtliche Untersuchungsergebnisse sind in Tab. 1 dargestellt. Durchschnittlich wurden bei markierten Varroa-Milben 1,73, bei unmarkierten 1,82 Deuto- 
nymphen pro fertilem Weibchem registriert. Für Drohnenbrut wurden 2,69 Deutonymphen ermittelt. Erhebliche Unterschiede zeigten die einzelnen Versuchsgruppen bezüglich des Anteils fertiler Milben : Während $95 \%$ aller in Drohnenbrut eingedrungener Tiere Nachkommen hatten, lag die Quote bei Arbeiterbrut mit unmarkierten Milben lediglich bei $73 \%$.

ТАВ. 1. - Vermehrungsraten von Varroa in Arbeiter- und Drohnenbrut

TABL. 1. - Reproductive rates of Varroa on drone brood and worker brood

\begin{tabular}{|c|c|c|c|c|c|c|}
\hline \multirow[b]{2}{*}{$\begin{array}{l}\text { Untersuchte Varroae } \\
\text { Examined Varroae }\end{array}$} & \multicolumn{2}{|c|}{$\begin{array}{l}\text { Markierte Milben } \\
\text { in Arbeiterbrut } \\
\text { Marked Varroae } \\
\text { in } \varnothing \text {-brood }\end{array}$} & \multicolumn{2}{|c|}{$\begin{array}{l}\text { Varroae in } \varnothing \text {-Brut } \\
\text { (natürlicher Befall) } \\
\text { Varroae in } \varnothing \text {-brood } \\
\text { (natural infection) }\end{array}$} & \multicolumn{2}{|c|}{$\begin{array}{l}\text { Varroae in } \delta \text {-Brut } \\
\text { (natürlicher Befall) } \\
\begin{array}{c}\text { Varroae in } \delta^{-} \text {-brood } \\
\text { (natural infection) }\end{array}\end{array}$} \\
\hline & 155 & $100 \%$ & 450 & $100 \%$ & 660 & $100 \%$ \\
\hline $\begin{array}{l}\text { Keine Reproduktion } \\
\text { No reproduction } \\
\text { Verspätete Eilage } \\
\text { To late in oviposition }\end{array}$ & 95 & 61 & $\begin{array}{l}72 \\
23\end{array}$ & $\begin{array}{l}16 \\
5\end{array}$ & & \\
\hline $\begin{array}{l}\text { Ohne lebensfähige Nachkommen } \\
\text { Without viable offspring }\end{array}$ & 103 & 66 & 95 & 21 & 24 & 4 \\
\hline $\begin{array}{l}\text { Nur } \sigma^{\pi} \text { Nachkommen } \\
\sigma^{\pi} \text { stages only }\end{array}$ & 7 & 5 & 27 & 6 & 8 & 1 \\
\hline $\begin{array}{l}\text { Mit } \subsetneq \text { Nachkommen } \\
\text { ๆ stages }\end{array}$ & 45 & 29 & 328 & 73 & 628 & 95 \\
\hline $\begin{array}{l}\text { Deutonymphen pro Varroa } \\
\text { Deutonymphs per Varroa } \\
1 \\
2 \\
3 \\
4 \\
5\end{array}$ & $\begin{array}{r}13 \\
31 \\
1 \\
0 \\
0\end{array}$ & & $\begin{array}{r}98 \\
191 \\
39 \\
0 \\
0\end{array}$ & & $\begin{array}{r}63 \\
184 \\
274 \\
99 \\
8\end{array}$ & \\
\hline $\begin{array}{l}\text { Durchschnittliche Anzahl } \\
\text { Deuton./Varroa } \\
\text { Average number deutonymphs per } \\
\text { Varroa }\end{array}$ & 1,73 & & 1,82 & & 2,69 & \\
\hline
\end{tabular}




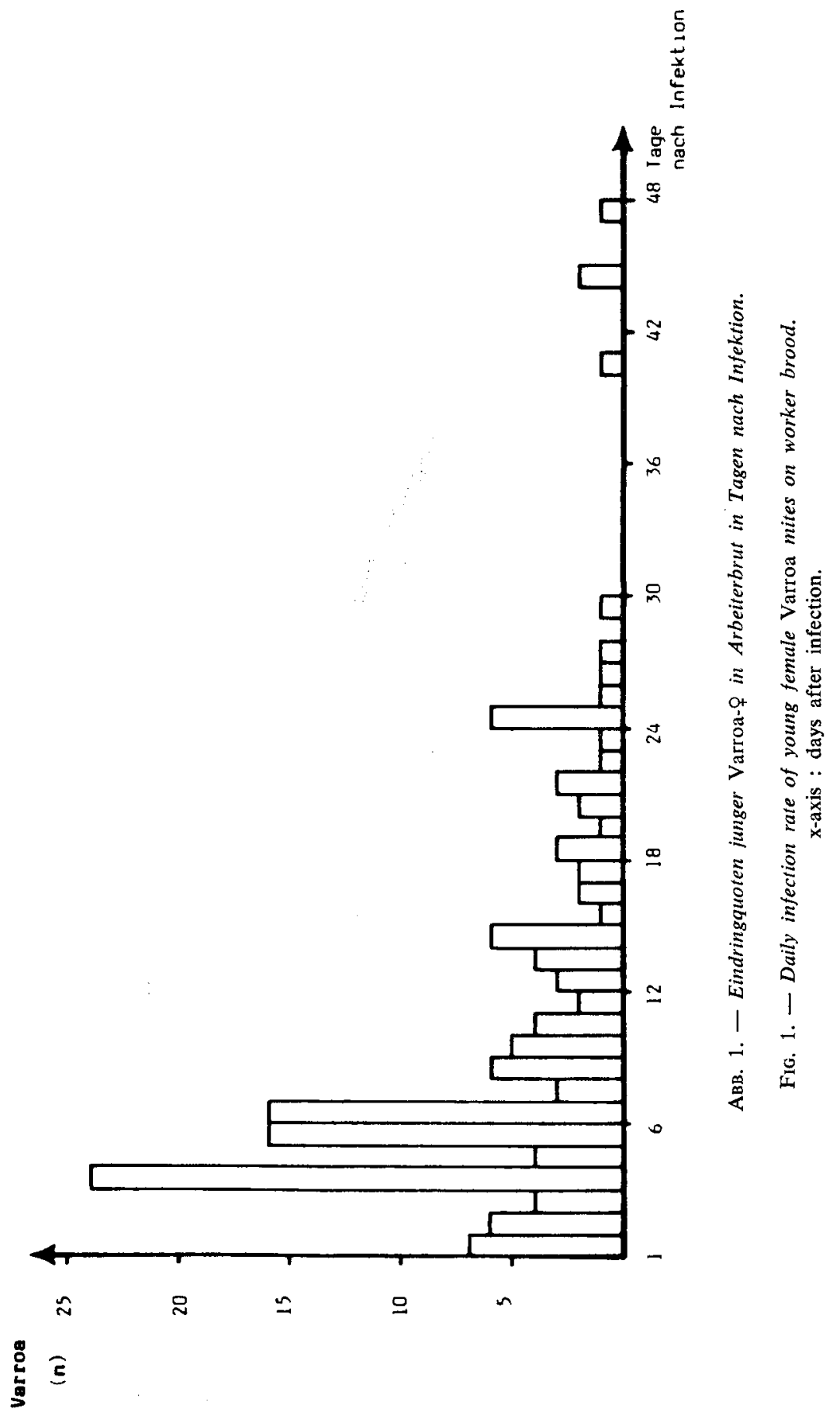


2) Frequenz der Reproduktionszyklen von Varroa

Von 78 markierten wiederholt eingesetzten Milben gingen $72(=92 \%)$ verloren bzw. starben im Volk. Lediglich $6(8 \%)$ Varroae drangen erneut in Brutzellen ein und nur 2 davon hatten erneut Nachkommen.

3) Zeitpunkt des Eindringens von Varroa in $\Varangle-B r u t$ unter kontrollierten Bedingungen

Die Befunde der einzelnen Versuchsansätze gehen aus den Abbildungen 1-3 hervor : Abb. 1) gibt die Verteilung von 142 am Schlupftag markierten und in die Versuchsvölker gesetzten Varroa-Milben wieder. Gemäß Abb. 2) dringen 43 Milben nach voraufgegangener Reproduktion wiederholt in ఫ-Brutzellen ein. Abb. 3) zeigt, im 6-Tage-Rhythmus aufgeteilt, die prozentuale Verteilung einer Gruppe von 298 Milben gemischter Altersstruktur.

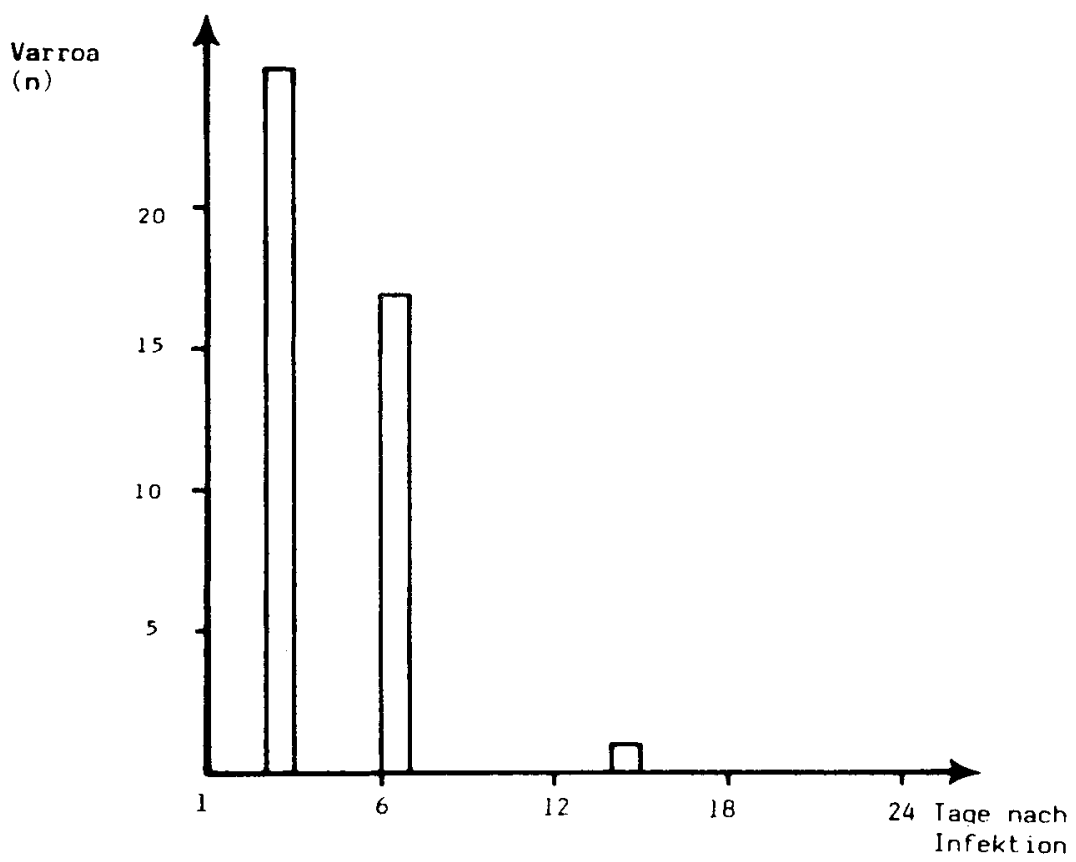

Aвв. 2. - Tägliche Eindringquoten von Varroa-«Müttern» in Arbeiterbrut zur wiederholten Reproduktion.

FIG 2. - Daily infection rate of female Varroae (《mother mites») on worker brood for repeated reproduction.

$\mathrm{x}$-axis : days after infection. 


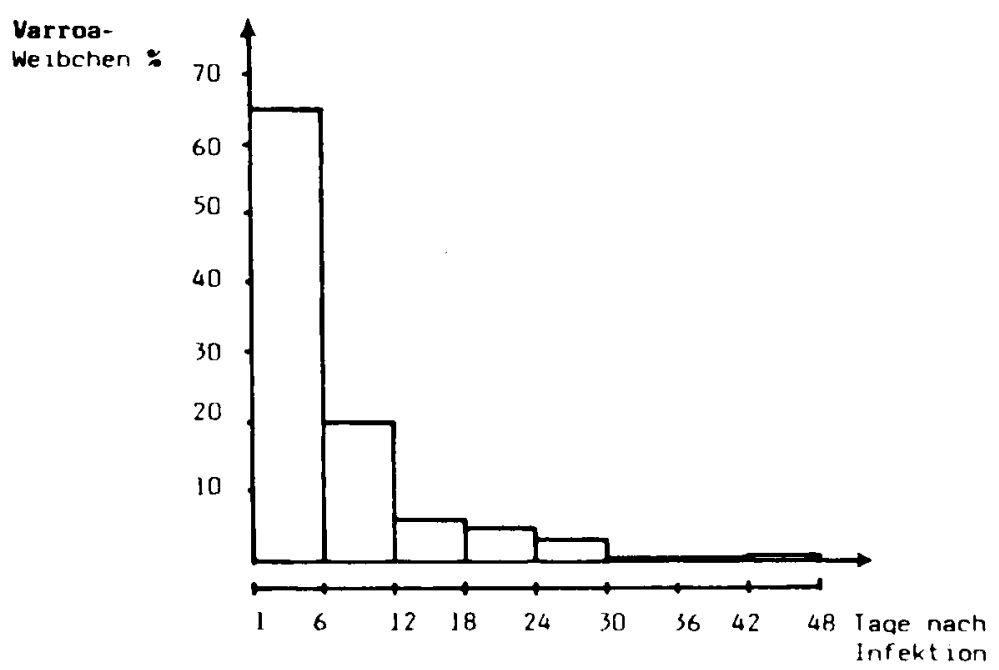

Aвв. 3. - Eindringquoten von Varroa-Populationen gemischter Aliersstruktur. Prozentuale Darstellung im 6-Tage Rhythmus.

Fig. 3. - Infection behaviour of mixed aged Varroa populations. Summarized in 6-day rhythms. $\mathrm{x}$-axis : days after infection; $\mathrm{y}$-axis : percent female Varroa in brood.

4) Abhängigkeit der Reproduktion des Parasiten vom Brutzyklus des Wirtes unter natïrlichen Bedingungen

Die bei Kontrolle von insgesamt 392557 Bienen ermittelten Milbenwerte sind in Tab. 2 zusammengestellt. Während der Trachtzeit (Mai-Juli) drangen innerhalb 24 Tage im Mittel 84.9\% $(\mathrm{s}=10,9 ; \mathrm{n}=15)$ aller im Versuchszeitraum registrierten Varroa-« Mütter $\gg(100 \%)$ in die Brut ein. Dic Auswertung der Restmilben ergab, daß3 durchschnittlich 4,8\% (s=3,8;n=15) Milben innerhalb 48 Tagen keine Brutzellen zur Reproduktion aufsuchen. Im Spätsommer (August-September) drangen innerhalb der beobachteten 24 Tage durchschnittlich $74,9 \%(s=2,6 ; n=4)$ in Arbeiterbrutzellen ein.

\section{5) Attraktivität von Arbeiter- und Drohnenbrut für infektionsbereite Milben}

Bei vergleichenden Stichprobenauszählungen von 13 Drohnen- und Arbeiterbrutproben wurde im arithmetischen Mittel ein bevorzugter Befall durch Varroa für Drohnenbrut vor Arbeiterbrut im Verhältnis 8,6:1 ermittelt (Tab. 3). 
TAB. 2. - Durch fortgesetzte Kontrolle sämtlicher Jungbienen registrierte Varroamilben («Muttermilben ») nach 24 und 48 Tagen

TABL. 2. - Total number of Varroae (mother mites) recorded by examination of all newly emerged bees after 24 and 48 days

\begin{tabular}{c|c|c|c|c}
\hline $\begin{array}{c}\text { Jahr } \\
\text { Year }\end{array}$ & $\begin{array}{c}\text { Volk } \\
\text { Colony }\end{array}$ & $\begin{array}{c}\text { Varroae n. 24 Tagen } \\
\text { Varroae after 24 days }\end{array}$ & $\%$ & $\begin{array}{c}\text { Varroae n. 48 Tagen } \\
\text { Varroae after 48 days }\end{array}$ \\
\hline
\end{tabular}

Gruppe a) Versuche Mai - Juli

Examinations May - July

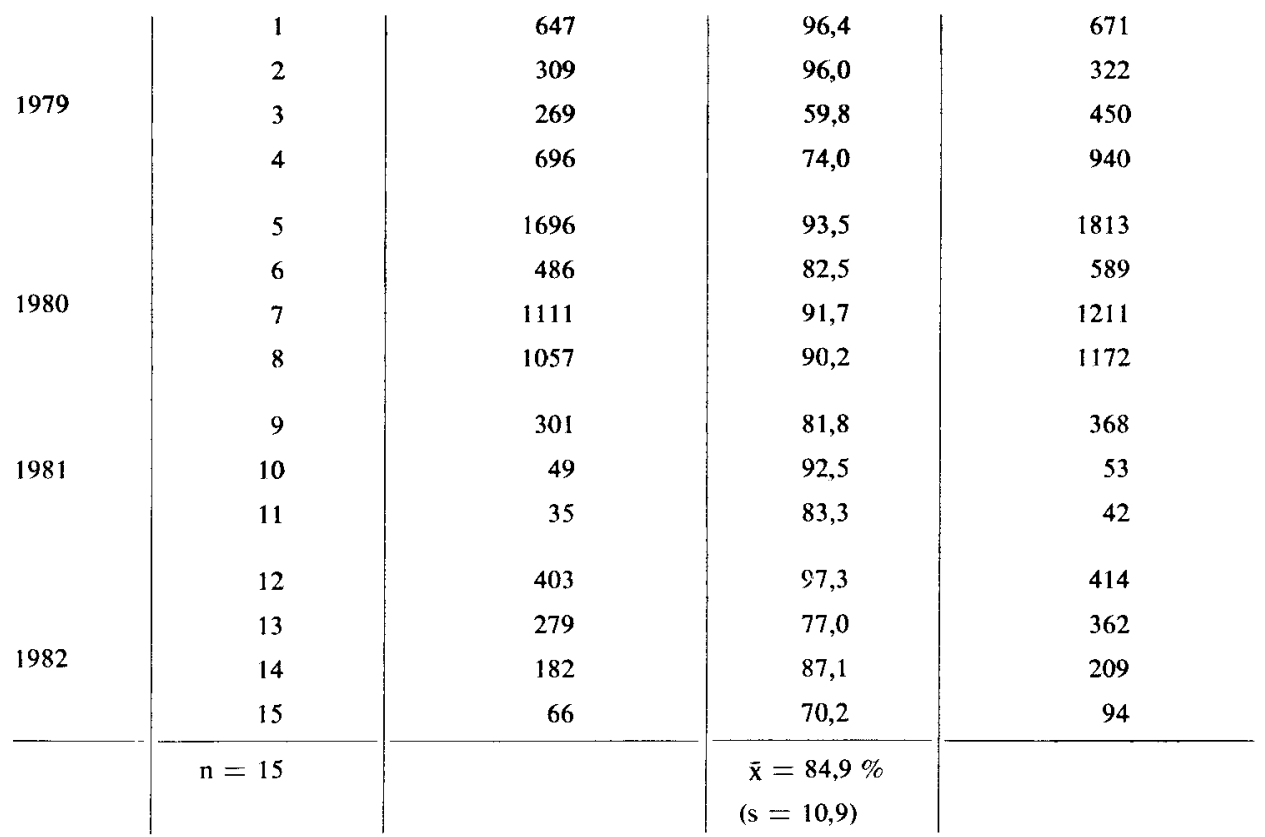

Gruppe b) Versuche August - September

Examinations August - September

\begin{tabular}{|c|c|c|c|c|}
\hline \multirow{5}{*}{1982} & 1 & 1536 & 74,1 & 2072 \\
\hline & 2 & 2463 & 73,9 & 3336 \\
\hline & 3 & 3859 & 73,0 & 5284 \\
\hline & 4 & 2641 & 78,7 & 3358 \\
\hline & $\mathrm{n}=4$ & & $\begin{array}{l}\bar{x}=74,9 \% \\
(s=2,6)\end{array}$ & \\
\hline
\end{tabular}




\section{DISKUSSION}

\section{1) Vermehrungsrate von Varroa in Arbeiter- und Drohnenbrut}

Die Angaben der Fachliteratur zur Reproduktion von Varroa sind häufig widersprüchlich. Die Aussagen hinsichtlich der pro Weibchen gelegten Eier schwanken zwischen 2 und 5 (SALTSCHENKO, 1972); durchschnittlich 5 Eier werden nach MuravsKaYa (1983) abgelegt; 7 bis 8 Eier gibt Grobov (1977) an. Nach Kamburov et al., 1975 und Mikitiuk et al., 1976, kann die Eizahl pro Eilage zwischen 1-2, sogar bis 38 Eier variieren. Es wird betont, daß nicht alle Milben die gleiche Anzahl Eier legen (Smirnow, 1979). Zudem soll die durchschnittliche Eizahl im Laufe der Brutsaison Schwankungen unterworfen und im Frühling gering sein, um ihr Maximum im Hoch- bzw. Spätsommer zu erreichen (Grobov, 1977). Diese Arbeiten lassen nicht erkennen, wie die genannten Daten ermittelt wurden, so daß sie unter Vorbehalt zu bewerten sind. Zudem häufen sich Befunde die dafür sprechen, daß die Varroatose auf den diversen Bienenrassen (ScherebKIN, 1978), wie unter verschiedenen Klimabedingungen (DE JoNG et al., 1983 ; MaUl et al., 1983), einen unterschiedlichen Verlauf nehmen kann. Somit muß SmIRNow (1979) beigepflichtet werden, der fordert, daß die Untersuchung der Biologie von Varroa und die Umstände der Parasitenentwicklung. in Abhängigkeit von der jeweiligen Zone zu erfolgen hat.

Wie Beobachtungen an Zellen mit schlupfreifer Brut zeigen, ist häufig mit einer Anzahl nicht ausdifferenzierter Nachkommen zu rechnen, so daß alleine auf Grund von Eizahlen noch keine Populationsdynamik zu betreiben ist. IFANTIDIS (1981) ermittelte, daß lediglich 58,5\% aller Milben bei Schlupf der Bienen das adulte Stadium erreicht haben. Nach seinen Befunden liegt die durchschnittliche Nachkommenzahl pro Gelege um 1. Bei Versuchen mit markierten VarroaMilben konnte ein Großteil (ca. $50 \%$ ) der über Jungbienen eingesetzten Milben nicht mehr registriert werden. Daneben drangen viele markierte Varroae in Zellen ein, ohne in Eilage zu gehen, bzw. verendeten dort (Tab. 1). Bei den beobachteten Ausfällen war mit einer verfahrensbedingten Verfälschung der Reproduktionsraten zu rechnen. Zur Absicherung wurden daher zusätzliche Untersuchungen an unmarkierten Milben aus natürlich befallener Arbeiterbrut angeschlossen. Aus zahlreichen Präparationen ist bekannt, daß sich häufig tote Milben ohne Nachkommen in den Zellen finden.

Bei unserem Untersuchungsmaterial war die Entscheidung, ob die Milben bereits vor der Tiefkühlung verendet waren, nicht zu treffen. Beobachtungen an markierten Altmilben zeigten, daß ein Großteil wieder eingedrungener Tiere keine Brutstadien aufwies. Somit läge die Vermutung nahe, daß es sich bei den $16 \%$ Milben, die nicht in Eilage gegangen waren, zumeist um Altmilben handelte. $\mathrm{Zu}$ spät in Eilage gegangen waren, wie im Markierungsversuch, $5 \%$ (23) aller Mil- 
ben. Ebenso gute Übereinstimmung mit den markierten Milben zeigte der Anteil Varroae, der nur männliche Nachkommen hervorbrachte mit $6 \%$ (27 Milben), gegenüber $5 \%$ (7 Tiere) im Markierungsversuch. Der Vergleich zwischen natürlich befallener und mit markierten Milben infizierter Arbeiterbrut in Bezug auf die Anzahl registrierter Deutonymphen ergab jedoch keinen statistisch gesicherten Unterschied $\left(\mathrm{chi}^{2}=4,33 ; \mathrm{p}=0,2\right)$. Bei Zusammenfassung der Ergebnisse aus beiden Untersuchungsreihen errechneten wir einen Mittelwert von $\overline{\mathrm{x}}=1,8$ ( $\mathrm{n}=373 ; \mathrm{s}=0,6071$ ) weiblicher Deutonymphen pro fertilem Varroa-Weibchen im Stadium Pd/Pdl (9., 10. Puppentag). Die Beobachtung, daß bei Schlupf varroainfizierter junger Arbeiterinnen häufig immature Milbenstadien registriert werden, legte die Vermutung nahe, daß die Verdeckelungszeit der Arbeiterzelle für die Entwicklung sämtlicher Varroabrut zu kurz sei, oder daß die Milben zu spät mit der Eiablage einsetzen. So hätte man annehmen können, daß bei ciner um 3 Tage längeren Verdeckelungszeit i.d.R. auch mit einer um ca. 3 Töchter höheren Nachkommensrate aus Drohnenbrut zu rechnen ist. Bei den untersuchten Zellen zeigte sich, daß bei einem Mittelwert von 2,7 Nachkommen pro Mutter mit einer nur um 0,9 höheren Reproduktionsrate gegenüber Arbeiterbrut zu rechnen ist ; womit die Verdeckelungsdauer der Drohnenzellen sicher nicht der einzig maßgebliche Faktor ist, der die höhere Anzahl Tochtermilben bedingt. Der bei präparierter Drohnenbrut registrierte Anteil Milben der nicht oder zu spät in Eilage gegangen war, betrug lediglich $4 \%$ ( 24 Milben). Ausschließlich männliche Nachkommen hatten nur $1 \%$ ( 8 Milben). $95 \%$ (628 Milben) hatten weibliche Nachkommen. In diesen Zahlen spiegelt sich die hervorragende Bedeutung der Drohnenbrut für die Varroa-Vermehrung wieder : Reproduktionsfähige Weibchen drängen zielstrebig in Drohnenzellen, während Arbeiterbrut in relativ hohem Maße nochmals von Altmilben infiziert wird, die nicht mehr in Reproduktion gehen und häufig in den Brutzellen verenden. Die gewonnenen Nachkommenswerte erfassen ausschließlich entwicklungsfähige Deutonymphen (9./10. Puppentag). Da diesen Stadien im Schutze der Zelle kaum mehr etwas zustoßen kann, wurde eine pauschale Schlupfquote von $100 \%$ angenommen.

Bezogen auf die Gesamtpopulation müssen die Vermehrungsquoten allerdings um die jeweils durchschnittlich beobachteten Ausfälle korrigiert werden, die sich bei Varroa-Milben in $\varnothing$-Brut auf ca. $26-27 \%$, bei $\delta$-Brut auf etwa $5 \%$ summieren. Unter diesen Bedingungen beziffert sich die durchschnittliche Reproduktionsrate für einen Vermehrungszyklus pro Varroa-Milbe auf :

1,3 Töchter in ఫ-Brut und

2,6 Töchter in $\sigma^{\prime}$-Brut.

Unter Berücksichtigung der Befunde zur Reproduktionsfrequenz (IV. 2) erhöht sich die gesamte Nachkommensquote im Leben einer Durchschnitts-Varroa auf :

1,6 Töchter in ఫ-Brut/bzw.

2,9-3,2 Töchter in $\sigma^{2}$-Brut. 


\section{2) Frequenz der Reproduktionszyklen von Varroa}

Unter Berücksichtigung der verfahrensbedingten Ausfälle in den Versuchen mit markierten Milben, zeigen unsere Ergebnisse gute Übereinstimmung mit den Angaben von Kamburov et al., 1975 und Mikitiuk et al., 1976, wonach $78 \%$ aller Milben einmal, $18 \%$ zweimal und $4 \%$ dreimal in Reproduktion gehen. Allerdings sind die Zahlen infolge der immensen Verluste zu niedrig für eine gesicherte Aussage.

\section{3) Zeitpunkt des Eindringens von Varroa in Arbeiter-Brut}

Bei einer von Langhe et al., 1977, angegebenen Lebensdauer der Sommermilben von 2 Monaten, wäre es möglich, daß die Fertilität der Varroa-Weibchen über diesen Zeitraum erhalten bleibt. Danach beginnt das Milbenweibchen im Alter von 5 Tagen mit der Ernährung und Eiablage.

Bei den von uns ermittelten 1,3 Töchtern pro Vermehrungsgang in ArbeiterBrut und einer durchschnittlichen Entwicklungsdauer von 8-9 Tagen (SALTSCHENKo, 1972), beginnt Varroa spätestens am 10.-11. Entwicklungstag der Bienenpuppe mit der Eilage. Die Infektion der Zelle erfolgt etwa am 7. Entwicklungstag. Somit scheint Langhe et al. das frühest mögliche Ereignis zu beschreiben. Nach Grobov (Zit. Smirnow, 1979) geht Varroa im Alter von 4-13 Tagen in Eilage.

Informationen, bis zu welchem Lebensalter eine Sommermilbe fertil bleibt, fehlen in der Literatur.

a) Das Alter der am Schlupftag markierten 142 Jungmilben wurde nach Angaben von IFANTIDIS (1981) zum Modus der Eiablage bei Varroa auf 2 Tage \pm 1 Tag festgesetzt. In Übereinstimmung mit den Angaben von LangHE et al. (1977) drangen bereits am Tage der Infektion $7(5 \%)$ Milben in Brutzellen ein (s. Abb. 1). Nach 4 Tagen wurde ein Maximum mit $24(17 \%)$ Individuen beobachtet. Es folgten zwei weitere Mamixa mit je $16(11 \%)$ eingedrungenen Varroae am 6. und 7. Tag nach Infektion. Bis zu diesem Zeitpunkt waren $55 \%$ aller Jungmilben in die Bienenbrutzellen gegangen. Bis zum 28. Tag wurde weiterhin der kontinuierliche Übertritt der Parasiten auf den Wirt registriert. Danach gingen bis zum 48. Tag nach Infektion noch vereinzelt Varroae in die Zellen. $\mathrm{Zu}$ einem späteren Zeitpunkt - beobachtet wurden bis 84 Tage nach Einsatz der markierten Versuchstiere - drang keine weitere Varroa-Milbe mehr in die Brut. Der verzögerte Übergang der Milben auf die Arbeiterbrut war in den Versuchen in keinem Fall auf zu geringes Brutzellenangebot $(>100$ Zellen pro reproduktionsfähige Varroa) zurückzuführen. Das ermittelte Durchschnittsalter der Milben bei Erstinfektion einer Bienenpuppe betrug ca. 13 Tage $(\bar{x}=12,7$ Tage, $n=140$, $\mathrm{s}=9,2) .90 \%$ aller beobachteten Jungmilben drangen innerhalb 24 Tagen in 
die Brutzellen ein. Von dieser Gruppe wurde ein Durchschnittsalter bei Befall der Zelle von 10,4 Tagen $(n=126, s=5,7)$ ermittelt. Es zeigte sich, daß Sommermilben maximal bis zum Alter von 50 Tagen mit der Infektion einer Bienenpuppe warten können.

b) An einer Vergleichsgruppe sollte das Reproduktionsverhalten nach voraufgegangener Eilage untersucht werden. Von ca. 350 markierten «Muttermilben » wurden 43 wieder in Brutzellen registriert (Abb. 2). Innerhalb 6 Tagen drangen 42 erneut in die Zellen ein, eine weitere Varroa folgte am 14. Tag nach Infektion. Nach diesen Befunden scheinen Varroa-Weibchen beim zweiten Befall rascher in die Zellen einzudringen als beim ersten.

c) Um die Situation eines von Varroatose betroffenen Bienenvolkes zu simulieren, wurde eine Versuchsgruppe mit gemischter Altersstruktur markiert und hinsichtlich ihres Eindringverhaltens beobachtet (Abb. 3). Auch durch diesen Versuchsansatz wurden die Ergebnisse der voraufgegangenen Testserien bestätigt. Danach drangen innerhalb 6 Tagen ca. $65 \%$ aller Milben in Arbeiterbrut ein. Nach 12 Tagen konnten bereits nahezu $85 \%$ und nach 24 Tagen $95 \%$ aller Milben in den Zellen registriert werden.

\section{4) Abhüngigkeit der Reproduktion des Parasiten vom Brutzyklus des Wirtes unter natürlichen Bedingungen}

$\mathrm{Da}$ das Untersuchungsmaterial aus Wirtschaftsvölkern mit natürlicher Varroa-Infektion bestand, wiesen die Völker die im Taunusgebiet beobachteten Streuungen auf. Die insgesamt rel. niedrigen Milbenzahlen in den Versuchen zur Trachtzeit 1981 und 1982 sind im Zusammenhang mit den voraufgegangenen Akarizidbehandlungen zu sehen (Winter 1980/1981 K-79-Feldversuch; Spätherbst 1981 Folbex VA-Neu).

Bei der angewandten Versuchsanordnung konnten im Verlauf von 21 Tagen nach Beginn der kontrollierten Bruterzeugung noch Jungbienen mit Varroa-Befall in den Völkern schlüpfen. Diese Milben waren bei Versuchsende zwischen 28 und 50 Tage alt und somit noch reproduktionsfähig. Nach unseren Befunden mit markierten Jungmilben wären allerdings bei Testende bereits mehr als $90 \%$ dieser Varroae in Brutzellen eingedrungen. Innerhalb 24 Tagen wurden ca. $85 \%$ aller in Brutzellen eingedrungener Milben registriert, was die Befunde der Markierungsversuche bestätigt.

Trotz unregelmäßiger Brutaktivitäten konnten innerhalb 48 Tagen von 15 Völkern, über die fortgesetzte Kontrolle sämtlicher Jungbienen, bei 2 Völkern $100 \%$, bei den übrigen zwischen $99,4 \%$ und $88,3 \%$ der Milben registriert werden. Im arithmetischen Mittel drangen ca. $95 \%$ aller Varroae im Versuchs- 
zeitraum Mai-Juli in die Arbeiterbrut ein, während ca. $5 \%$ über die Auswertung der Restbienen ermittelt wurde. Bei den Versuchen im August-September (1982) wurden aus Gründen der saisonbedingt nachlassenden Brutaktivitäten der Völker lediglich 4 Kontrollserien untersucht. Unter der Annahme- in Analogie o.g. Befunde-, daß von der Gesamtmilbenpopulation $95 \%$ in Brutzellen eindringen, wurde aus den Brutkontrolldaten der Anteil «Muttermilben » hochgerechnet und die Quote der innerhalb 24 Tagen in Brutzellen registrierten Tiere bestimmt. Wir errechneten, daß im Spätsommer lediglich $75 \%$ Milben gegenüber $85 \%$ im Hochsommer in die Brut eindrangen. Damit konnte gegenüber der Haupttrachtzeit ein verzögertes Reproduktionsverhalten von Varroa nachgewiesen werden. Im weiteren wurden die ermittelten Milbenquoten auf ihre Abhängigkeit vom jeweiligen Brutzellenangebot analysiert. Aus Daten von 15 Völkern (Versuche MaiJuli, 1979-1982) wurde über die Methode der «Kleinsten quadratischen Abweichung » (minimum least square) für einen Zeitraum von je 6 Tagen der durchschnittlich zu erwartende Anteil reproduktionswilliger Varroa-Weibchen bestimmt. Danach würden innerhalb 6 Tagen maximal $57 \%(\mathrm{~s}=35,6353)$ einer natürlichen Milbenpopulation die Bienenbrut infizieren. Die für die Versuchsvölker berechneten theoretischen Erwartungswerte (SOLL) wurden mit den Versuchsdaten (IST) verglichen und zum ermittelten Brutzellenangebot in Beziehung gesetzt (BIENEN/SOLL).

Abb. 4 gibt die Befunde graphisch wieder. Der Kurvenlauf der Funktion $[y=a+b \ln (x)]$ wird bestimmt durch die Regressionskoeffizienten $a=0,43$ und $b=0,09$. Die logarithmische Kurvenanpassung ergab mit einem Korrelationskoeffizienten von $\mathrm{r}^{2}=0,43(\mathrm{p}=0,01)$ eine signifikant bessere Näherung als die lineare Regressionsgerade.

\section{5) Attraktivitüt von Arbeiter- und Drohnenbrut für infektionsbereite Milben}

Die Befunde der Stichprobenerhebungen im Monat Mai (Tab. 3) beweisen die deutliche Bevorzugung der Drohnenbrut vor Arbeiterbrut. Bei den größtenteils stark befallenen Völkern zeigten sich allerdings extreme Schwankungen die noch eine endguiltige Bewertung erschweren. Der errechnete Attraktivitätsfaktor von 8,6: 1 für Drohnnbrut befindet sich in guter Übereinstimmung mit Sulimanovic et al. (1982), die ein Verhältnis von 7,2: 1 mitteilen. 


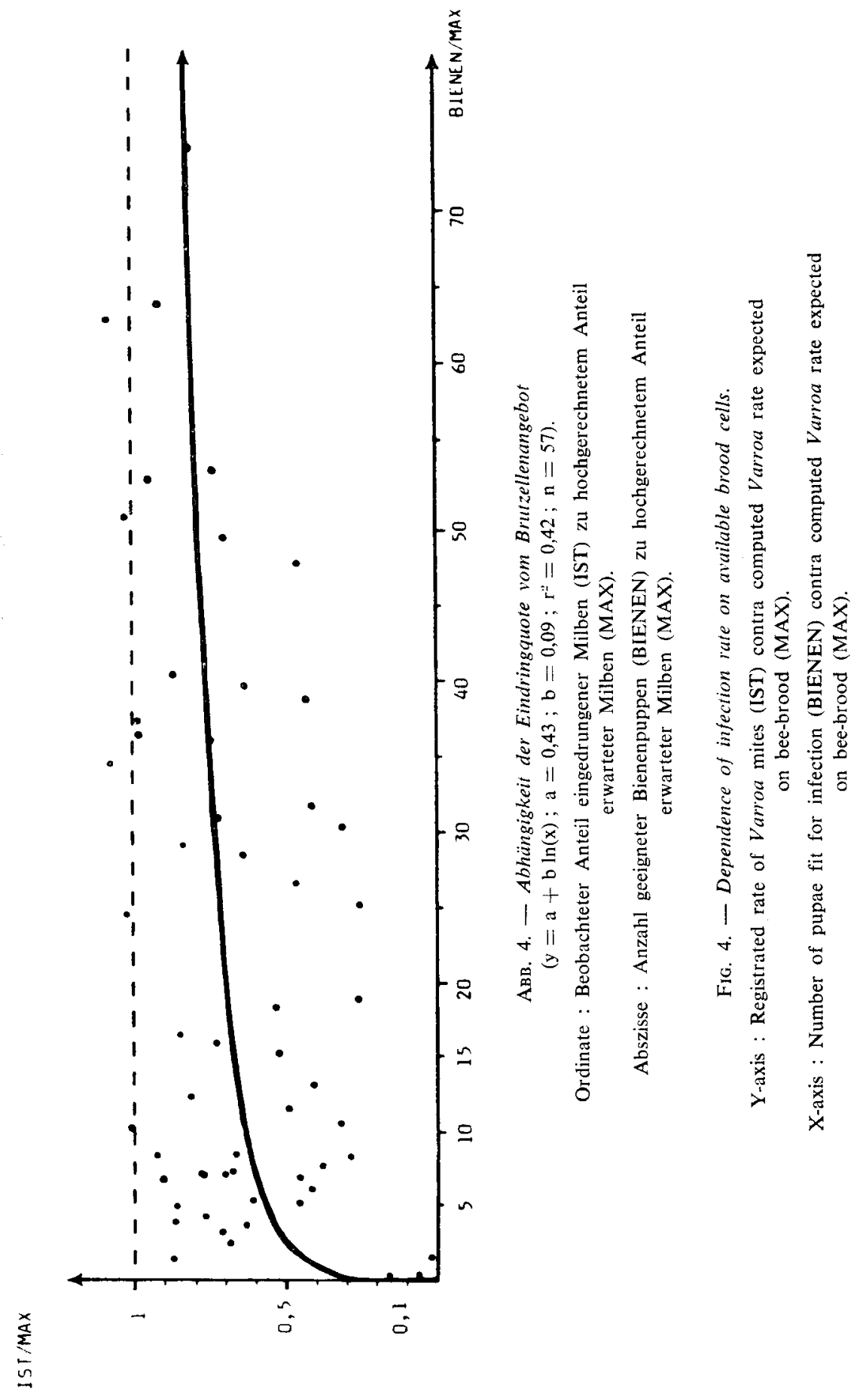




\title{
DANKSAGUNG
}

Danken möchte ich all jenen, die zum Gelingen der Untersuchungen beigetragen haben.

Namentlich : Herrn Prof. Dr. N. Koeniger für die Vergabe des Themas und die Betreuung der Arbeit; besonders Herrn Prof. Dr. F. Rutrner für seine vielfältige Unterstützung; sowie Herrn Dr. R. MORITZ für seine Hilfe bei den Computerauswertungen. Zu Dank verpflichtet bin ich der DFG für die finanzielle Förderung der Arbeit.

\author{
RÉSUMÉ \\ REPRODUCTION ET DYNAMIQUE DES POPULATIONS DE LACARIEN \\ PARASITE VARROA JACOBSONI OUD. EN RELATION AVEC LE CYCLE \\ DE SON HOTE, APIS MELLIFICA L.
}

(1' PARTIE)

On a démarré cette recherche afin d'obtenir des données sur le comportement de reproduction de Varroa. On a étudié a) du couvain d'ouvrières et de mâles infesté naturellement, b) du couvain d'ouvrières infesté avec des acariens marqués individuellement, pour éclaircir les points suivants : tous les combien Varroa se reproduit-il ? à quel âge les acariens commencent-ils à se reproduire? quel est le taux de multiplication de Varroa?

Pour le marquage, nous avons utilisé une colle au cyanacrylate mélangé à un pigment fluorescent. Le mélange a été appliqué à l'aide d'une aiguille spéciale $(\varnothing: 0,2 \mathrm{~mm})$ sur le scutellum dorsal d'acariens non anesthésiés. Pour reconstituer les dates d'infestation, on a produit des séries de couvain : la reine a été maintenue sur 3 cadres à couvain vides placés entre des cadres de provisions. Tous les 6 jours nous ôtions les cadres contenant le couvain et en remettions des vides. Le couvain était placé dans une hausse de la ruche jusqu'au $18^{\circ}$ jour. Il était alors enlevé et placé en étuve $\left(34^{\circ} \mathrm{C}, 70 \%\right.$ d'humidité). On a déterminé l'infestation par Varroa en contrôlant toutes les abeilles fraîchement écloses ou en ouvrant les cellules de couvain avant l'éclosion.

Nous avons déterminé une moyenne de 1,73 deutonymphes femelles par Varroa fertile dans les séries avec les acariens marqués et de 1,82 chez le couvain infesté naturellement (moyenne globale : 1,8) (Tabl. 1). Avec le couvain de mâles on a obtenu en moyenne 2,7 deutonymphes femelles par Varroa. En conditions naturelles, nous avons trouvé $73 \%$ d'acariens fertiles dans le couvain d'ouvrières, mais $95 \%$ dans le couvain de mâles. On a pu démontrer qu'environ $78 \%$ des acariens se reproduisaient une fois et $22 \%$ deux fois. Sur la population totale de Varroa, le taux de reproduction global est de 1,6 descendants femelles (adultes) sur couvain d'ouvrières et de 2,9-3,2 sur le couvain de mâles. Les acariens Varroa préfèrent le couvain d'ouvrières par rapport au couvain de mâles dans la proportion de 8,6:1 au mois de mai (Tabl. 2). Les taux journaliers d'infestation ont été analysés dans les conditions optimales d'élevage (infestation $<1 \%$ ) (Figs 1,2 et 3). Les acariens qui s'étaient déjà reproduits détectaient plus rapidement les cellules hôtes que les jeunes acariens (Figs 1, 2). En été les acariens peuvent rester fertiles pendant 50 jours. $95 \%$ de la population totale d'acariens (tous âges confondus) ont pénétré les cellules du couvain en 24 jours. Une expérience semblable a été menée sur des colonies exploitées en conditions naturelles ayant des quantités de couvain variables (Tabl. 3 et 4). Les résultats concordent avec ceux trouvés au laboratoire. La méthode du carré minimum a montré que $57 \%$ de la population de Varroa essayaient d'infester les nymphes d'abeille dans les 6 jours. Cette proportion dépend de la quantité de couvain disponible (Fig. 4). 


\section{SUMMARY \\ REPRODUCTION AND POPULATION DYNAMICS OF THE PARASITIC MITE VARROA JACOBSONI OUD. AND ITS DEPENDENCE ON THE BROOD CYCLE OF ITS HOST APIS MELLIFERA L.}

This investigation was started to obtain data on the reproductive behaviour of Varroa. We examined : a) naturally infested worker and drone brood and b) worker brood infested with individually marked mites. Then we asked a) how often is Varroa reproductive, b) how old are the mites when they start reproduction, c) what is the multiplication rate of Varroa.

For marking we used a mixture of cyanacrylate and a fluorescent pigment. We applied this on the dorsal scutellum of the unnarcotized mite with the help of a special needle (Minutie, $0.2 \mathrm{~mm}$ ). To establish the infection dates, we produced series of bee brood : the queen was restricted to three empty brood-combs between food-combs. In 6-day cycles we took off the frames containing the brood and added new empty ones. The brood was set into the honey compartment of the colony until the 18 th day. Then it was taken off and set into an incubator $\left(34^{\circ} \mathrm{C}, 70 \%\right.$ humidity). For determining Varroa infestation we controlled all freshly emerged bees or onened the brood cells before emergence.

We counted an average of 1.73 female deutonymphs per fertile Varroa in the series with marked mites and an average of 1.82 in naturally infested worker brood (Total average $=1.8$ ) (Tabl. 1). In drone brood we counted and average of 2.7 female offspring (deutonymphs) per Varroa. Under natural conditons we found $73 \%$ fertile mites in worker brood, but $95 \%$ fertile Varroa in drone brood. $78 \%$ reproduced only once and $22 \%$ twice. For the whole Varroa population we found a total reproductive rate on worker brood of about 1.6 adult daughters and on drone brood 2.9-3.2.

Varroa mites prefer drone brood instead of worker brood by $8.6: 1$ in the month of May (Tabl. 2). The daily infection rates were observed under optimal breeding conditions (infestation Varroa : bees was 1:100) (Fig. 1, 2, 3). Mites of a parental generation entered brood cells more quickly than young mites for the first reproductive cycle (Fig, 1, 2). Mites in summer remain fertile for 50 days. $95 \%$ of the whole Varroa population occurred in brood cells during 24 days.

A similar experiment was started with common bee colonies under natural conditions and various breeding situations (Tabl. 3, 4). The results are in good accordance with those found in laboratory. Using the least-squares method, we found $57 \%$ of the Viarroa population try to infest the bee pupae within 6 days. This depends on the supply of suitable brood cells (Fig. 4).

\section{LITERATUR}

De Jong D., Morse R.A., Gonçalves L.S., 1983. - Regional differences in the virulence of Varroa jacobsoni (abstract). Third int. Symp. on Invertebrate Reproduction, Tübingen.

Grobov O.F., 1977. - Varroasis in bees. In : Varroasis, a honeybee disease. Bukarest, Apimondia Publ. House, 46-70.

IfaNTIDIS M.D., 1981. - Versuche zur kontrollierten Aufzucht von Varroa Milben. In : Diagnose und Therapie der Varroatose. Bukarest, Apimondia Verlag, 32-38.

JAY S.C., 1962. - Colour changes in honeybee pupae. Bee World, 43, 119-122.

Kamburov G. et al., 1975. - Zit. nach Grobov O. (1977), a.a. O.

Koeniger N., Schulz A., 1980. - Versuche zur biologischen Therapie der Varroatose durch eine Kontrolle der frisch geschlüpften Bienen. Apidologie, 11 (2), 105-112. 
Langhe A.B., Natzki K.V., Natzkil V.M., 1977. - The mite Varroa and the methods of controlling it. In : Varroasis, a honeybee disease. Bucharest, Apimondia Publ. House,

Maul V., Kiepsch A., Wachendörfer G., 1983. - Befallstärke der Varroatose in Hessen im Herbst 1982. Die Biene, (5), 197-199.

Mikitiuk V.V. et al., 1976. - Zit. nach Grobov O. (1977), a.a. O.

MuraVskaya A.J., 1982. - Assessment of the reproductive capacity of female Varroa jacobsoni (in Russian). Veterinariya, Moscow (2), 49-54 [AA, 1983, 34 (1)].

Ritter W., Ruttner F., 1980. - Diagnoseverfahren. Allg. dtsch. Imkerzig., (5), 134-138.

SALTSChenko V.L., 1966-1972. - Zit. nach Grobov O. (1977), a.a. O.

Smirnow A.M., 1979. - Aktuelle Ergebnisse der sowjetischen Wissenschaft über Ätiologie, Pathogenie, Epizootologie, Diagnose und Bekämpfung der Varroatose. In : Bekämpfung und Vorbeugung der Varroatose. Bukarest, Apimondia-Verlag, 60-74.

Smirnow O., 1979. - Über die Morphologie und Histologie der Milbe Varroa jacobsoni. In : Bekämpfung und Vorbeugung der Varroatose. Bukarest, Apimondia-Verlag, 36-40.

Sulimanovic D., Ruttner F., Pechiacker H., 1982. - Studies on the biology of reproduction in Varroa jacobsoni (in Japanese). Honeybee Sci., 3 (3), 109-112.

Scherebrin, 1978. - Zit. nach Smirnow O. (1978), 67 a.a. O. 